\title{
CANONICAL EQUATIONS FOR A CONSTRAINED MECHANICAL SYSTEM
}

\author{
DO SANH \\ Hanoi Technology University
}

\section{§1. INTRODUCTION}

In many theoretical studies it is convenient to transform Lagrange's equations to the canonical form where the canonical variables are introduced for substuting the Lagrange's ones. It is a set of $2 n$ variables $\left\{q_{i}, p_{i}\right\}(i=\overline{1, n})$ and in these variables the motion of a system is described by $2 n$ ordinary differential equations of the first order.

First, as known the canonical equations was established for a conservative holomonic mechanical system. Later a similar form was expended for a nonconservative mechanical system and nextly, for a nonholonomic system (the form of canonical equations with undefined multipliers) [2, $3,8]$. However, the above mentioned estsblished form of canonical equations haven't many practice senses.

In the present work the author proposes a form of canonical equations for a constrained mechanical system applying usefully for holonomic and nonholonomic systems. These equations are constructed by the help of the principle of compatibility [1]. Such a form of canonical equations will be used comfortable for studying dynamic of a multibody system.

\section{§2. CANONICAL EQUATIONS FOR A CONSTRAINED MECHANICAL SYSTEM}

Let us consider a holonomic mechanical system. The position of the system is defined by Lagrange's coordinates $q_{i}(i=\overline{1, n})$. There exists a force function $U$ of active forces.

Hamilton reduced the differential equations of motion to a very significant form called the Hamilton canonical equations.

For the aim of establishing canonical equations, instead of variables $\dot{q}_{i}$ we introduced new variables $p_{i}(i=\overline{1, n})$, that is:

$$
p_{i}=\frac{\partial T}{\partial \dot{q}_{i}}
$$

where $T$ is the kinetic energy of the system which is assumed to be positive define quadratic form.

The variables $p_{i}$ are known as impulses and are conjugates of the Lagrange's coordinates. Since the highest order of form with respect to $\dot{q}_{i}$ in the expression for the kinetic energy, the transform form $\dot{q}_{i}$ to $p_{i}$ is one-to-one.

Let us now introduce the Hamilton function:

$$
H=\sum_{i=1}^{t} \dot{q}_{i} p_{i}-T-U=H\left(t, q_{i}, p_{i}\right)
$$


where the variables $\dot{q}_{i}$ are expressed through the impulses $p_{i}$ by the help of the transformation (2.1). By such a way the Hamilton function is the function of canonical variables $\left\{q_{i}, p_{i}\right\}(i=\overline{1, n})$.

As known the equations of motion of the unconstrained system can be written in the form

$$
\begin{array}{ll}
\dot{q}_{i}=\frac{\partial H}{\partial p_{i}} & (i=\overline{1, n}) \\
p_{i}=-\frac{\partial H}{\partial q_{i}} & (i=\overline{1, n})
\end{array}
$$

where the group of equations (2.3) expresses the transformation of variables (2.1), and the group of equations (2.4) describes the motion of the unconstrained system.

Let us now consider the mechanical system subjected to the nonholonomic constraints of the form

$$
\sum b_{\alpha i} \dot{q}_{i}+b_{\alpha}=0 \quad(\alpha=\overline{1, r} ; r<n)
$$

where $b_{\alpha i}=b_{\alpha i}\left(t, q_{j}\right) ; b_{\alpha}=b_{\alpha}\left(t, q_{i}\right)$ are known functions.

In taking account of the transformation (2.1), the equation (2.5) can be written in the following form

$$
\sum B_{\alpha_{i}} p_{i}+b_{\alpha}=0
$$

where

$$
B_{\alpha i}=\sum_{j=1}^{n} b_{\alpha j} a_{j i}
$$

$\left\|a_{i j}\right\|$ is the inverse matrix of the matrix of inertia, which is $n \times n$ positive define symmetric matrix, too.

Evidently, the equations (2.4) can't describe the motion of the system with constraints (2.5). or in the equivalent form (2.6).

To write the equations of motion of the system with constraints (2.5) or in the equivalent form (2.6) we will apply the principle of compatibility [1].

Of course, the first group of equations (2.3) treated as a transformation of variables can be keep in tact, and the second group of the equations of motion must be substitited by the following equations

$$
p_{i}=-\frac{\partial H}{\partial q_{i}}+R_{i}
$$

where $R_{i}$ are the reactions of the constraints (2.5) or in the equivalent form (2.6), which by the principle of compatibility must satisfy the algebraic equations

$$
\sum_{i=1}^{n} B_{\alpha i} R_{i}+B_{\alpha}^{0}=0
$$

where

$$
B_{\alpha i}^{0}=b_{\alpha}-\sum_{i=1}^{n} B_{\alpha i} \frac{\partial H}{\partial q_{i}}
$$

which are the functions of canonical variables $\left\{q_{i}, p_{i}\right\}(i=\bar{i}, n)$. 
As a result we obtain $(2 n+r)$ equations $(2.3),(2.7)$ and $(2.8)$ which contain $3 n$ unknowns $q_{i}$, $p_{i}, R_{i}(i=\overline{1, n})$.

Because of $r<n$, the number of obtained equations is smaller than the one of unknowns, therefore the problem is underfined. The above mentioned nondeterminate is caused by the substance of the considered constraints. This means that it is necessary to know the information about the substance of considered constraints. In connection with this, let us consider a class of ideal constraints. Following [1], the condition of ideality of the constraints (2.5) can be written in the form

$$
\sum_{i=1}^{n} d_{\nu i} R_{i}=0 \quad(\nu \pm \overline{1, k} ; k=n-r)
$$

where $d_{\nu i}$ are the coefficients in the expressions of generalized accelerations $\ddot{q}_{i}$ when they are represented through independent accelerations by the present of constraints (2.5). $(i=\overline{1}, n)$.

By such a way we obtain $3 n$ equations $(2.3),(2.4),(2.9)$ and $(2.11)$ of $3 n$ unknowns $q_{i}, p_{i}, R_{i}$

In general, the set of above mentioned equations will give the unique solution $\left\{q_{i}(t), p_{i}(t), R_{i}(t)\right\}$ which describe the motion of the considered system together with the reactions of constraints $(2.5)$ acting upon the system.

For the aim of applying the numerical methods to solve the problem of dynamics of multibody system, let us introduce their matrix notations, for example, $q$ is the notations of the $1 \times n$ line matrix, but $\underline{q}$ - its transform matrix.

We have then

$$
\begin{gathered}
\dot{q}=\underline{A} \underline{p} \\
\underline{p}=-\frac{\partial H}{\partial \underline{q}}+\underline{R} \\
\underline{B} \underline{R}+\underline{B}^{0}=0 \\
D \underline{R}=0
\end{gathered}
$$

where $\underline{A}$ is the inverse matrix of the matrix of inertia, which is the $n \times n$ positive define symmetric matrix too.

$$
\begin{aligned}
\underline{q} & =\left\|q_{1} q_{2} \ldots q_{n}\right\|^{T} \\
\underline{p} & =\left\|p_{1} p_{2} \ldots p_{n}\right\|^{T} ; \\
\underline{R} & =\left\|R_{1} R_{2} \ldots R_{n}\right\|^{T}-\text { the matrix of constaint reactions } \\
\underline{B} & =\underline{b}_{1} \underline{A}
\end{aligned}
$$

$\underline{b}_{1}$ is the $r \times n$ matrix of the elements of the form $\left\|b_{\alpha i}\right\|$ Besides

$$
B^{0}=\left\|b_{\alpha}-\sum B_{\alpha i} \frac{\partial H}{\partial q_{i}}\right\|
$$

is the $n \times 1$ matrix and

$$
\underline{D}=\left\|d_{\nu i}\right\|
$$

is the $k \times n$ matrix, the elements of which are determined by identities

$$
\underline{b}_{1} \underline{D}=0
$$




\section{§3. EXAMPLES}

Example 1. Two ponderable partides $M_{1}$ and $M_{2}$ of identical mass $m=1$ are jointed by a rod of constant lenght $\ell$ and neglegibly small mass. The system is constrained to move in the horizontal plane and only in such manner that the velocity of midpoint of the rod is directed along it. Write the canonical equations of the system.

Let $x_{1}, y_{1}$ and $x_{2}, y_{2}$ 'be the coordinates of the partides $M_{1}$ and $M_{2}$. We introduce the following generalized coordinates $q_{1}=x_{1}+x_{2} ; q_{2}=x_{1}-x_{2} ; q_{3}=y_{1}+y_{2} ; q_{4}=y_{1}-y_{2}$.

It is easy to see that the constraint equations may be written as follows

$$
\begin{aligned}
& \ddot{q}_{2} \dot{q}_{3}-q_{4} \dot{q}_{1}=0 \\
& q_{2}^{2}+q_{4}^{2}-\ell^{2}=0
\end{aligned}
$$

The last equations can be written in the equivalent form

$$
q_{2} \dot{q}_{2}+\dot{q}_{4} \dot{q}_{4}=0
$$

The kinetic energy of the system will be now

$$
T=\frac{1}{2}\left(\dot{q}_{1}^{2}+\dot{q}_{2}^{2}+\dot{q}_{3}^{2}+\dot{q}_{4}^{2}\right)
$$

The matrix of inertia is the unique matrix. Therefore its inverse matrix is the unique one, too.

It is easy to calculate the following matrices

$$
\begin{gathered}
\underline{B}=\left\|\begin{array}{cccc}
0 & q_{2} & 0 & q_{4} \\
-q_{4} & 0 & q_{2} & 0
\end{array}\right\| \\
\underline{p}=\left\|\begin{array}{cccc}
1 & 0 & q_{4} / q_{2} & 0 \\
0 & 1 & 0 & -q_{2} / q_{4}
\end{array}\right\| \\
\underline{B}^{0}=\left\|\begin{array}{c}
p_{1}^{2}+p_{4}^{2} \\
p_{2} p_{3}-p_{4} p_{1}
\end{array}\right\|
\end{gathered}
$$

Equations of motion will be

$$
\begin{aligned}
& \dot{q}_{1}=p_{1} ; \quad \dot{q}_{2}=p_{2} ; \quad \dot{q}_{3}=p_{3} ; \quad \dot{q}_{4}=p_{4} \\
& \dot{p}_{1}=R_{1} ; \quad \dot{p}_{2}=R_{2} ; \quad \dot{p}_{3}=R_{3} ; \quad \dot{p}_{4}=R_{4} \\
& q_{2} R_{2}+q_{4} R_{4}+p_{2}^{2}+p_{4}^{2}=0 ; \\
& \quad-q_{4} R_{1}+q_{2} R_{3}+p_{2} p_{3}-p_{4} p_{1}=0 \\
& R_{1}+\frac{q_{4}}{q_{2}} R_{3}=0 ; \quad R_{2}-\frac{q_{2}}{q_{4}} R_{4}=0
\end{aligned}
$$

Example 2 [8]. Let us consider the motion of a platform in an inclined plane (Fig. 1). We reduce it to fixed rectangular coordinates $O X Y Z$. Assume that $x, y$ are the coordinates of the contact point, $a$ - the distance from the contact point to the mass centre of the platform. The wheel is treated as a thin knife.

The kinetic energy and potential function are

$$
\begin{aligned}
& T=\frac{1}{2}\left[\dot{x}^{2}+\dot{y}^{2}+\rho^{2} \dot{\varphi}^{2}+2 a \dot{\varphi}(\dot{y} \cos \varphi-\dot{x} \sin \varphi)\right] \\
& U=-m g x \sin \alpha
\end{aligned}
$$

where $\rho$ is the radius of inertia of the platform with respect to the mass centre. 


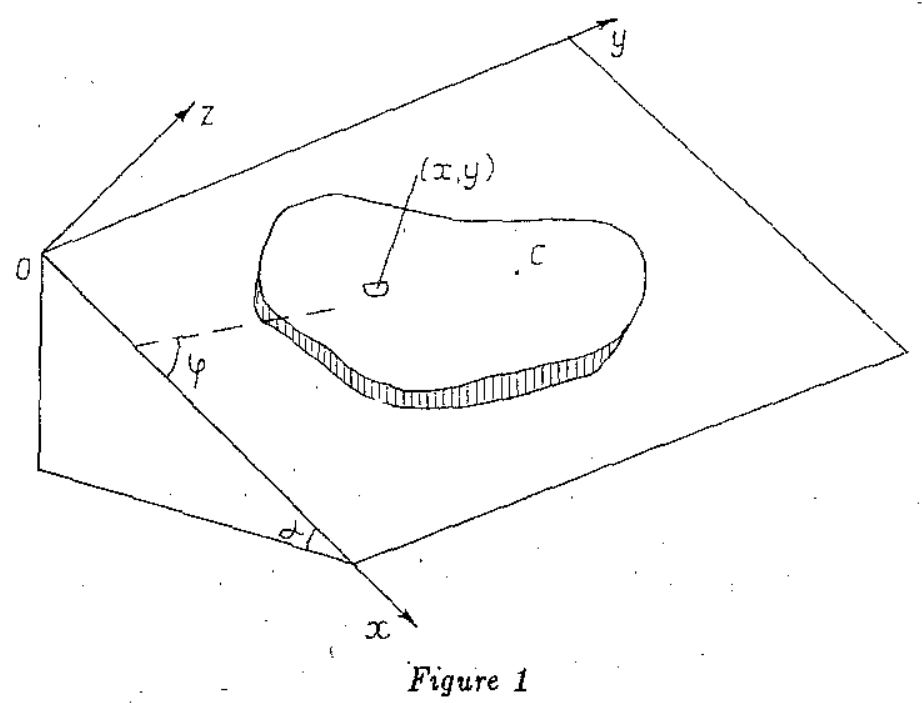

The constraint equations can be written as follows

$$
\dot{x} \operatorname{tg} \varphi-\dot{y}=0
$$

The matrix of inertia has the form

$$
\underline{A}^{*}=\left\|\begin{array}{ccc}
m & 0 & -m a \sin \varphi \\
0 & m & m a \cos \varphi \\
-m a \sin \varphi & m a \cos \varphi & m \rho^{2}
\end{array}\right\|
$$

The inverse matrix of the matrix of inertia will be

$$
\underline{A}=\frac{1}{m\left(\rho^{2}-a^{2}\right)}\left\|\begin{array}{ccc}
\rho^{2}-a^{2} \cos ^{2} \varphi & -a^{2} \sin \varphi \cos \varphi & a \sin \varphi \\
-a^{2} \sin \varphi \cos \varphi & \rho^{2}-a^{2} \sin ^{2} \varphi & -a \cos \varphi \\
a \sin \varphi & -a \cos \varphi & 1
\end{array}\right\|
$$

Besides, we have

$$
\begin{aligned}
& \underline{B}=\left\|\rho^{2} \sin \varphi \quad-\rho^{2} \cos \varphi \quad a \cos \varphi\right\| \\
& \underline{B}^{0}=\left\|\left(a^{2} \sin \varphi \cos \varphi\left(p_{x}^{2}-p_{y}^{2}\right)-a^{2} \cos 2 \varphi p_{x} p_{y}+a \cos \varphi p_{x} p_{\varphi}+a \sin \varphi p_{y} p_{\varphi}\right)\right\| \\
& \underline{D}=\left\|\begin{array}{ccc}
1 & \operatorname{tg} \varphi & 0 \\
0 & 0 & 1
\end{array}\right\|
\end{aligned}
$$

Equations $\left(2.3^{\prime}\right)$ and $\left(2.8^{\prime}\right)$ take the form

$$
\begin{aligned}
\dot{x} & =\frac{1}{m\left(\rho^{2}-a^{2}\right)}\left[\left(\rho^{2}-a^{2} \cos ^{2} \varphi\right) p_{x}-a^{2} \sin \varphi \cos \varphi p_{y}+a \sin \varphi p_{\varphi}\right] \\
\dot{y} & =\frac{1}{m\left(\rho^{2}-a^{2}\right)}\left[-a^{2} \sin \varphi \cos \varphi p_{x}+\left(\rho^{2}-a^{2} \sin ^{2} \varphi\right) p_{y}-a \cos \varphi p_{\varphi}\right] \\
\dot{\varphi} & =\frac{1}{m\left(\rho^{2}-a^{2}\right)}\left[a \sin \varphi p_{x}-a \cos \varphi p_{y}+p_{\varphi}\right] \\
\dot{p}_{x} & =R_{x} \\
\dot{p}_{y} & =R_{y} \\
\dot{p}_{\varphi} & =-\frac{1}{2} a^{2} \sin 2 \varphi\left(p_{x}^{2}-p_{y}^{2}\right)+a^{2} \cos 2 \varphi p_{x} p_{y}-a \cos p_{x} p_{\varphi}-a \sin \varphi p_{y} p_{\varphi}+R_{\varphi}
\end{aligned}
$$


Equations (2.9') and (2.11') are now

$$
\begin{aligned}
& \rho^{2} \sin \varphi R_{x}-\rho^{2} \cos \varphi R_{y}+a \cos \varphi R_{\varphi}-a^{2} \cos \varphi \sin \varphi\left(p_{x}^{2}-p_{y}^{2}\right)- \\
& -a^{2} \cos 2 \varphi p_{x} p_{y}+a \cos \varphi p_{x} p_{\varphi}+a \sin \varphi p_{y} p_{\varphi}=0 \\
& R_{x}+R_{y} \operatorname{tg} \varphi=0 ; \quad R_{\varphi}=0
\end{aligned}
$$

\section{§4. CONCLUSION}

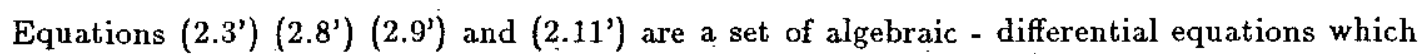
permit to determine simultaneously the motion of the system and the constraint reactions subjected upon the system. We have obtained two of important results

To obtain the form of canonical equations for constrained mechanical systems.

The canonical equations are written in the matrix notation, which are convenient.for applying methods of computation mechanics to solve problems of multibody system dynamics.

This publication is completed with financial support from the National Basic Research Program in Natural Sciences.

\section{REFERENCES}

1. Do Sanh. Motion of a constrained mechanical system. Thesis of Doctor of Sciences, Hanoi Thechnology University, Hanoi 1984:

2. Goldstein H. Classical mechanics, Addision - Wesly Reading, Mass 1950.

3. Haar D. Ter. Elements of Hamiltonian mechanics, University Reader in Theoretical Physics, Oxford.

4. Kamman N. and Huston R. L. Dynamics of constrained multibody systems, ASME Journal of Applied Mechanics, Vol. 51, No 41988.

5. Kane T. R. and Levinsion D. A. Multibody Dynamics, ASME Journal of Applied Mechanics, Vol. 50, 1983.

6. Kane T. R. and Levinsion D. A. Dynamics theory and applications, Mc Graw-Hill, New York, 1985.

7. Lurie A. I. Analytical Mechanics, Moscow, 1961 (in Russian).

8. Nejmark J. I. and Fufajew N. A. Dynamics of nonholonomic system, “Nauka”, Moscow, 1967 (in Russian).

9. Chetaev N. G. Theoretical mechanics, Mir Publishers, Moscow, 1989.

Received December 91,1999

\section{PHUOONG TRÌNH CHÍNH TĂC CHO CƠ HỆ CHỊU LIÊN KẾT}

Trong công trình đã xây dựng hệ phương trình chính tắc cho một cơ hệ chịu liên kết. Đây là hệ $2 n$ phương trình đại số cấp một. Đã nhận được 2 kết quá quan trọng:

Nhận được một dạng các phương trình chính tắc cho các cơ hệ chịu liên kết.

Các phương trình được viết ớ dạng ma trận rất thích hợp cho việc áp dụng các phương pháp của cơ học tính toán để giải các bài toán động lực học hệ nhiều vật. 